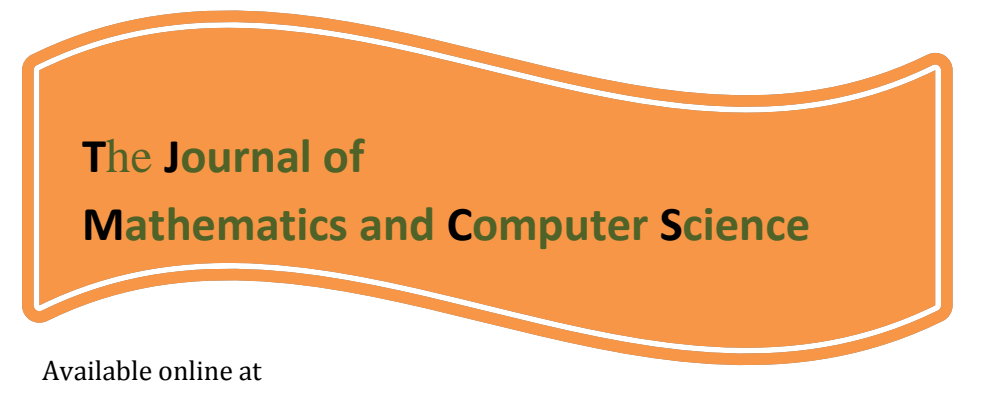

http://www.TIMCS.com

The Journal of Mathematics and Computer Science Vol .3 No.1 (2011) 21-34

\title{
On behavior of preconditioned methods for a class of compact finite difference schemes in solution of hyperbolic equations
}

\section{A. Golbabai ${ }^{* 1}$ and M.M. Arabshahi ${ }^{2}$}

Department of Applied Mathematics, Faculty of Mathematics, Iran University of Science and Technology, Narmak, Tehran 16844, Iran

1golbabai@iust.ac.ir ,

2molavi@iust.ac.ir

Received: March 2011, Revised: May 2011

Online Publication: July 2011

\begin{abstract}
:
In this article, We apply Krylov subspace methods in combination of the ADI, BLAGE,... method as a preconditioner for a class of linear systems arising from compact finite difference schemes in solution of hyperbolic equations $\alpha \mathrm{u}_{\mathrm{tt}}-\beta(\mathrm{x}, \mathrm{t}) \mathrm{u}_{\mathrm{xx}}=\mathrm{F}\left(\mathrm{x}, \mathrm{t}, \mathrm{u}_{\mathrm{u}} \mathrm{u}_{\mathrm{x}}, \mathrm{u}_{\mathrm{t}}\right)$ subject to appropriate initial and Dirichlet boundary conditions, where $\alpha$ is constant. We show The BLAGE preconditioner is extremely effective in achieving optimal convergence rates. Numerical results performed on model problem to confirm the efficiency of our approach.
\end{abstract}

Keywords: Compact finite difference; Hyperbolic equations; Krylov subspace methods; Preconditioner.

Mathematics Subject Classifications: 65M06, 35L10

*Corresponding Author 


\section{1-Introduction:}

When solving PDE's by means of numerical methods one often has to deal with large systems of linear equations, especially if the PDE's is time-independent or if the timeintegrator is implicit [8]. For real life problems, these large systems can often only be solved by means of some iterative method. Even if the system is preconditioned, the basic iterative method often converges slowly or even diverges. The numerical solution of one space second order hyperbolic equations with nonlinear first derivative terms in Cartesian, cylindrical and spherical coordinates are of great importance in many fields of engineering and sciences. Many computational models give rise to large sparse linear systems. For such systems iterative methods are usually preferred to direct methods which are expensive both in memory and computing requirements. When the iterative method is based on Krylov subspaces, there is a need to use preconditioning techniques in order to achieve convergence in a reasonable number of iteration steps. Since the preconditioner plays a critical role in preconditioned Krylov subspace methods, many preconditioners have been proposed and studied [22, 5, 11]. Unfortunately, some preconditioners have been proposed and studied by many of researchers $[17,10,11]$, that are not effective for discretization of compact approximation. The ADI method is a preconditioner $[15,12]$ for non-symmetric systems that can be very effective but this method is not effective for more general block tri-diagonal systems arising from the high-order approximations. Also, BLAGE method [3] is proposed as a preconditioner for a class of non-symmetric linear systems arising from the high-order finite difference schemes. In this article, we compare different preconditioned methods for solving linear systems arising from the compact high-order approximation of hyperbolic equation

$$
\alpha \mathrm{u}_{\mathrm{tt}}-\beta(\mathrm{x}, \mathrm{t}) \mathrm{u}_{\mathrm{xx}}=\mathrm{F}\left(\mathrm{x}, \mathrm{t}, \mathrm{u}, \mathrm{u}_{\mathrm{x}}, \mathrm{u}_{\mathrm{t}}\right)
$$

Defined in the region $\mathrm{W} \times[0<\mathrm{t}<\mathrm{T}]$, where $\mathrm{W}=\{\mathrm{x} \mid 0<\mathrm{x}<1\}$ and $\alpha$ is constant. The initial conditions consists of

$$
\mathrm{u}(\mathrm{x}, 0)=\mathrm{g}_{1}(\mathrm{x}), \quad \mathrm{u}_{\mathrm{t}}(\mathrm{x}, 0)=\mathrm{g}_{2}(\mathrm{x}), \quad 0 \leq \mathrm{x} \leq 1,
$$

and boundary conditions consists of

$$
\mathrm{u}(0, \mathrm{t})=\mathrm{h}_{0}(\mathrm{t}), \quad \mathrm{u}(1, \mathrm{t})=\mathrm{h}_{1}(\mathrm{t}), \quad \mathrm{t} \geq 0,
$$

where $\mathrm{u}=\mathrm{u}(\mathrm{x}, \mathrm{t})$. The resulting block tri-diagonal linear system of equations is solved by using Krylov subspace methods. The outline of this paper is as follows: 
In Section 2, we briefly introduce some available preconditioners. In Section 3, we describe Krylov subspace methods. In Section 4 we present a class of compact highorder finite difference operators and in Section 5, we present an example arising from the compact high-order approximations. In Section 6, we report a brief conclusion.

\section{2-Preconditioner}

The convergence rate of iterative methods depends on spectral properties of the coefficient matrix. Hence we will attempt to transform the linear system into another equivalent system in the sense that it has the same solution, but has more favorable spectral properties. A preconditioner is a matrix that effects such as a transformation [2, 4]. If the preconditioner be as $M=M_{1} M_{2}$ then the preconditioned system is as

$$
\mathrm{M}_{1}^{-1} \mathrm{AM}_{2}^{-1}\left(\mathrm{M}_{2} \mathrm{x}\right)=\mathrm{M}_{1}^{-1} \mathrm{~b} \quad(2.1) .
$$

The matrices $M_{1}$ and $M_{2}$ are called the left and right preconditioners, respectively. Now, we briefly describe preconditioners that we use for solving linear systems and let us take A matrix arising from fourth order approximations that is block tri-diagonal.

\section{2-1Preconditioner based on relaxation technique}

Let $A=D+L+U$ such that $D, L$ and $U$ are diagonal, lower and upper triangular block matrices, respectively. A splitting of the coefficient matrix is as $A=M-N$ where the stationary iteration for solving a linear system is as

$$
\mathrm{x}^{(\mathrm{k}+1)}=\mathrm{M}^{-1} \mathrm{~N} \mathrm{x}^{(\mathrm{k})}+\mathrm{M}^{-1} \mathrm{~b}
$$

In Table 1, we briefly show preconditioners based on relaxation technique.

In the above notation, $\omega$ is called the relaxation parameter. The optimal value of the parameter $\omega$ reduces the number of iterations to a lower order [1]. We have chose M in Jacobi, G-S, SOR as a left preconditioner and in SSOR preconditioner, we have chose $\mathbf{M}_{1}=\frac{1}{\omega(2-\omega)}(\mathrm{D}+\omega \mathrm{L})$ as a left preconditioner and $\mathrm{M}_{2}=\mathrm{D}^{-1}(\mathrm{D}+\omega \mathrm{U})$ as a right preconditioner. Also, we take $\omega_{o p t}=\frac{2}{1+\sqrt{1-\rho_{J}^{2}}}$ 
A. Golbabai and M.M. Arabshahi/ TJMCS Vol .3 No.1 (2011) 21-34

Table 1: Preconditioners based on relaxation technique Preconditioner

\begin{tabular}{|c|c|}
\hline Preconditioner & $\mathrm{M}$ \\
\hline Jacobi & $\mathrm{D}$ \\
\hline Gauss-Seidel & $(\mathrm{D}+\mathrm{L})$ \\
\hline SOR & $\frac{1}{\omega}(D+\omega L)$ \\
\hline SSOR & $\frac{1}{\omega(2-\omega)}(D+\omega L) D^{-1}(D+\omega U)$ \\
\hline
\end{tabular}

\section{2-2ADI preconditioner}

Peaceman and Rachford [16] in 1955 presented the ADI method for solving linear systems. Let $\mathrm{A}=\mathrm{H}+\mathrm{V}$ and in the form

$$
A=\left(\begin{array}{ccccc}
B_{1} & C_{1} & & & \\
A_{2} & B_{2} & C_{2} & & \\
& \ddots & \ddots & \ddots & \\
& & A_{n-1} & B_{n-1} & C_{n-1} \\
& & & A_{n} & B_{n}
\end{array}\right)
$$

Where $A_{i}=\operatorname{tridiag}\left\{a_{1 i}, b_{1 i}, c_{1 i}\right\}, \quad B_{i}=\operatorname{tridiag}\left\{a_{2 i}, b_{2 i}, c_{2 i}\right\} \quad$ and $C_{i}=\operatorname{tridiag}\left\{a_{3 i}, b_{3 i}, c_{3 i}\right\} \quad$ of order $N \times N$ where $\mathrm{H}$ and $\mathrm{V}$ are bounded and include $H=\left\{0.5 B_{i}, b_{3 i}, b_{1 i}\right\}$, $V=\left\{0.5 B_{i}, a_{1 i}, c_{1 i}, a_{3 i}, c_{3 i}\right\}$. The alternative direction implicit method for solving the linear system $A x=b$ is in following form:

$$
\begin{gathered}
\left(\mathrm{H}+\mathrm{r}_{1} \mathrm{I}\right) \mathrm{u}^{(\mathrm{k}+1 / 2)}=\mathrm{b}-\left(\mathrm{V}-\mathrm{r}_{1} \mathrm{I}\right) \mathrm{u}^{(\mathrm{k})}, \\
\left(\mathrm{V}+\mathrm{r}_{2} \mathrm{I}\right) \mathrm{u}^{(\mathrm{k}+1)}=\mathrm{b}-\left(\mathrm{H}-\mathrm{r}_{2} \mathrm{I}\right) \mathrm{u}^{(\mathrm{k}+1 / 2)},
\end{gathered}
$$

The ADI preconditioner is as $\mathrm{M}=\left(\mathrm{H}+\mathrm{r}_{1} \mathrm{I}\right)\left(\mathrm{V}+\mathrm{r}_{2} \mathrm{I}\right)$ that $\mathrm{M}_{1}=\left(\mathrm{H}+\mathrm{r}_{1} \mathrm{I}\right)$ and $\mathrm{M}_{2}=\left(\mathrm{V}+\mathrm{r}_{2} \mathrm{I}\right)$ where Parameters $r_{1}$ and $r_{2}$ are acceleration parameters. Young and Varga $[25,23]$ proved that the optimum value for $r_{1}$ and $r_{2}$ is $\sqrt{\alpha \beta}$ where $\alpha \leq \mu_{\mathrm{i}}, v_{\mathrm{i}} \leq \beta$ and $\mu_{i}, v_{i}$ are eigenvalues of matrices $\mathrm{H}$ and $\mathrm{V}$ respectively.

\section{2-3BLAGE preconditioner}

The BLAGE method [3, 7] was originally introduced as analogue of the AGE method [6]. The BLAGE uses fractional splitting technique that is applied in two half steps on linear systems with block tri-diagonal matrices of order $\mathrm{N}^{2} \times \mathrm{N}^{2}$ and in the form 


$$
A=\left(\begin{array}{ccccc}
B_{1} & C_{1} & & & \\
A_{2} & B_{2} & C_{2} & & \\
& \ddots & \ddots & \ddots & \\
& & A_{n-1} & B_{n-1} & C_{n-1} \\
& & & A_{n} & B_{n}
\end{array}\right)
$$

where $\mathrm{A}_{\mathrm{i}}, \mathrm{B}_{\mathrm{i}}$ and $C_{i}$ are tri-diagonal matrices of order $N \times N$. The splitting of matrix A is sum of matrices $G_{1}$ and $G_{2}$ in which $\mathrm{A}=\mathrm{G}_{1}+\mathrm{G}_{2}$ where $G_{1}$ and $G_{2}$ are of the form

$$
G_{1}=\left(\begin{array}{cccccc}
B_{1}^{\prime} & & & & & \\
& B_{2}^{\prime} & C_{2} & & & \\
& A_{3} & B_{3}^{\prime} & & & \\
& & & \ddots & & \\
& & & & B_{n-1}^{\prime} & C_{n-1} \\
& & & & A_{n} & B_{n}^{\prime}
\end{array}\right) \text { and } G_{2}=\left(\begin{array}{cccccc}
B_{1}^{\prime} & C_{1} & & & & \\
A_{2} & B_{2}^{\prime} & & & & \\
& & \ddots & & & \\
& & B_{n-2}^{\prime} & C_{n-2} & \\
& & & A_{n-1} & B_{n-1}^{\prime} & \\
& & & & & B_{n}^{\prime}
\end{array}\right)
$$

for odd values of $n$ where $B_{i}^{\prime}=\frac{1}{2} B_{i}$. The BLAGE preconditioner is as

$\mathrm{M}=\left(\mathrm{G}_{1}+\omega_{1} \mathrm{I}\right)\left(\mathrm{G}_{2}+\omega_{2} \mathrm{I}\right)$ that $\mathrm{M}_{1}=\left(\mathrm{G}_{1}+\omega_{1} \mathrm{I}\right)$ and $\mathrm{M}_{2}=\left(\mathrm{G}_{2}+\omega_{2} \mathrm{I}\right)$ where $\omega_{1}$ and $\omega_{2}$ are optimal iteration parameters. We have experimentally chosen the relaxation parameter $\omega_{1}=\sqrt{\alpha_{1} \beta_{2}}$ and $\omega_{2}=\sqrt{\alpha_{2} \beta_{1}}$ where $\alpha_{1}=\lambda_{\text {min }}\left(\mathbf{M}_{1}\right), \beta_{1}=\lambda_{\text {max }}\left(M_{1}\right)$ and $\alpha_{2}=\lambda_{\text {min }}\left(\mathbf{M}_{2}\right)$, $\beta_{2}=\lambda_{\max }\left(\mathrm{M}_{2}\right)$ so that we will have the minimum condition number.

\section{3-Krylov subspace methods}

Let $x_{0}$ be an arbitrary initial guess for linear systems given by $A x=b$ and let $\mathrm{r}_{0}=\mathrm{b}-\mathrm{Ax}_{0}$ be the corresponding residual vector. A Krylov subspace of order $m$ that is shown with $\mathrm{K}_{\mathrm{m}}(\mathrm{A}, \mathrm{r})$ is defined as follows:

$$
\mathrm{K}_{\mathrm{m}}\left(\mathrm{A}, \mathrm{r}_{0}\right)=\operatorname{span}\left\{\mathrm{r}_{0}, \mathrm{Ar}_{0}, \ldots, \mathrm{A}^{\mathrm{m}-1} \mathrm{r}_{0}\right\}
$$

For un-symmetric matrix A, different Krylov methods can be used such as GMRES, GMRES(m), QMR, CGS, BiCG, BiCGSTAB $[18,24]$. Now, we briefly describe some Krylov subspace methods:

\section{3-1Generalized Minimal residual(GMRES) method}

In 1986, Saad and Schultz [19] introduced GMRES method for solving non-symmetric systems. This method has the property of minimizing the norm of the residual vector over the Krylov subspace method at every step. The major drawback for GMRES method is that the amounts of work and storage required per iteration linearly rises with the 
iteration number. The usual way for overcome this problem is to restart after $\mathrm{m}$ iteration.

Proposition 3.1: Assume that $A$ is a diagonalizable matrix and let $A=\mathrm{XDX}^{-1}$ where $\mathrm{D}=\operatorname{diag}\left\{\lambda_{1}, \ldots, \lambda_{\mathrm{n}}\right\}$ is the diagonal matrix of eigenvalues. Define,

$$
\varepsilon^{(m)}=\min _{\mathrm{p} \in \mathrm{P}_{\mathrm{m}} \mathrm{p}(0)=1} \max _{\mathrm{i}=1, \ldots, \mathrm{n}}\left|\mathrm{p}\left(\lambda_{\mathrm{i}}\right)\right| \text {. }
$$

Then, the residual norm achieved by the m-th step of GMRES satisfies the inequality $\left\|r_{m}\right\| \leq K(X) \varepsilon^{m}\left\|\mathrm{r}_{0}\right\|_{2}$, Where $\mathrm{K}(\mathrm{X})=\|X\|_{2}\left\|X^{-1}\right\|_{2}$. When $A$ is positive real with symmetric part $M$, the following error bound can be derived from the proposition,

$$
\left\|r_{m}\right\| \leq[1-\alpha / \beta]^{m / 2}\left\|\mathrm{r}_{0}\right\|
$$

with $\alpha=\left(\lambda_{\min }(\mathrm{M})\right)^{2}, \beta=\lambda_{\max }\left(\mathrm{A}^{\mathrm{T}} \mathrm{A}\right)$. This proves the convergence of the GMRES(m) for all $m$ when $A$ is positive real [18].

\section{3-2Bi-Conjugate Gradient (BiCG) method}

Bi-conjugate gradient (BiCG) method was suggested by Fletcher in 1977, is applied to non-symmetric matrices. BiCG method needs matrix-vector products with $A$ and $A^{T}$. Also, BiCG method is sensitive to possible breakdowns and numerical instabilities Proposition 3.2: The vectors produced by the Bi-conjugate Gradient algorithm satisfy the following orthogonality properties:

$$
\begin{array}{lll}
\left(r_{j}, r_{i}^{*}\right)=0, & \text { for } & i \neq j, \\
\left(A p_{j}, p_{i}^{*}\right)=0, & \text { for } & i \neq j,
\end{array}
$$

The following theorem is well-known, [18].

\section{3-3Quasi- Minimal Residual (QMR) method}

In 1991, Freund and Nachtigal proposed the quasi-minimal residual (QMR) method for solving non-Hermitian linear systems. Later in 1994, they presented QMR method based on the coupled two-term recurrences instead of three-term recurrences [9]. This method sometimes avoids the break down of BiCG method. Also, QMR method has a regular convergence behavior than other Krylov subspace methods.

Proposition 3.3: The residual norm of the approximate solution $x_{m}$ of QMR method satisfies the relation $\left\|b-A x_{m}\right\| \leq\left\|V_{m+1}\right\|_{2} \mid s_{1} \ldots s_{m}\left\|r_{0}\right\|_{2}$.

The following theorem is well-known, cf. [18].

\section{3-4Conjugate Gradient Squared (CGS) method}


In 1989, Sonneveld presented the conjugate gradient squared (CGS) method for nonsymmetric systems [21]. The speed of convergence of this method usually is about twice as fast as BiCG method. Convergence behavior of this method is often quite irregular, which may result loss of accuracy in the updated residual. Algorithm of Preconditioned Conjugate Gradient Squared method is presented in [21].

\section{3-5Bi-Conjugate Gradient Stabilized (BiCGSTAB) method}

This method is applied for non-symmetric systems. Bi-conjugate gradient stabilized method is an alternative for CGS method that avoids the irregular convergence behavior of CGS method while maintaining about the same speed of convergence [20]. Algorithm of BiCGSTAB method that applied to the preconditioned system (2.1) is presented in [2].

\section{4-Compact high-order approximations}

Now let us $p=\frac{k}{h}$, Mohanty et al. [14] have derived finite difference schemes of fourthorder accuracy for equations of the form

$$
\mathrm{u}_{\mathrm{tt}}-\mathrm{A}(\mathrm{x}, \mathrm{t}) \mathrm{u}_{\mathrm{xx}}=\mu(\mathrm{x}, \mathrm{t}) \mathrm{u}_{\mathrm{x}}+v(\mathrm{x}, \mathrm{t}) \mathrm{u}_{\mathrm{t}}+\lambda(\mathrm{x}, \mathrm{t}) \mathrm{u}+\mathrm{f}(\mathrm{x}, \mathrm{t}),
$$

Here, we present the compact high-order scheme for (4.1) that can be written in the form

$$
\begin{aligned}
& \left(\lambda_{1}-T_{i+1}^{k+1}\right) u_{i+1}^{k+1}+\left(\lambda_{2}-T_{i-1}^{k+1}\right) u_{i-1}^{k+1}+\left(\lambda_{3}-T_{i}^{k+1}\right) u_{i}^{k+1}+\left(\lambda_{4}-T_{i+1}^{k}\right) u_{i+1}^{k}+\left(\lambda_{5}-T_{i-1}^{k}\right) u_{i-1}^{k} \\
& +\left(\lambda_{6}-T_{i}^{k}\right) u_{i}^{k+1}+\left(\lambda_{7}-T_{i+1}^{k-1}\right) u_{i+1}^{k-1}+\left(\lambda_{8}-T_{i-1}^{k-1}\right) u_{i-1}^{k-1}+\left(\lambda_{9}-T_{i}^{k-1}\right) u_{i}^{k-1}= \\
& \left.\frac{k^{2}}{2}\left[\left(r_{1}+8 h a_{1} Q_{i}^{k}\right) K_{i+1}^{k}+\left(r_{2}-8 h a_{1} Q_{i}^{k}\right) K_{i-1}^{k}+\left(1+8 k a_{2} R_{i}^{k}\right) K_{i}^{k+1}+\left(1-8 k a_{2} R_{i}^{k}\right) K_{i}^{k-1}+8 K_{i}^{k}\right)\right] .
\end{aligned}
$$

Where

$$
\begin{gathered}
T_{i+1}^{k+1}=4 k p^{2} b_{2} R_{i}^{k}+4 h b_{1} Q_{i}^{k}+\frac{k}{4} R_{i+1}^{k}\left(r_{1}+8 h a_{1} Q_{i}^{k}\right)+\frac{k p}{4} Q_{i}^{k+1}\left(1+8 h a_{2} R_{i}^{k}\right), \\
T_{i-1}^{k+1}=4 k p^{2} b_{2} R_{i}^{k}-4 h b_{1} Q_{i}^{k}+\frac{k}{4} R_{i-1}^{k}\left(r_{2}-8 h a_{1} Q_{i}^{k}\right)-\frac{k p}{4} Q_{i}^{k+1}\left(1+8 h a_{2} R_{i}^{k}\right), \\
T_{i+1}^{k-1}=-4 k p^{2} b_{2} R_{i}^{k}+4 h b_{1} Q_{i}^{k}-\frac{k}{4} R_{i+1}^{k}\left(r_{1}+8 h a_{1} Q_{i}^{k}\right)+\frac{k p}{4} Q_{i}^{k-1}\left(1-8 h a_{2} R_{i}^{k}\right), \\
T_{i-1}^{k-1}=-4 k p^{2} b_{2} R_{i}^{k}-4 h b_{1} Q_{i}^{k}-\frac{k}{4} R_{i-1}^{k}\left(r_{1}+8 h a_{1} Q_{i}^{k}\right)-\frac{k p}{4} Q_{i}^{k-1}\left(1-8 h a_{2} R_{i}^{k}\right), \\
T_{i+1}^{k}=2 k p Q_{i}^{k}+4 k^{2} c_{1} Q_{i}^{k}-8 h b_{1} Q_{i}^{k}+\frac{k^{2}}{2} S_{i+1}^{k}\left(r_{1}+8 h a_{1} Q_{i}^{k}\right) \\
+\frac{3 k}{4} p Q_{i+1}^{k}\left(r_{1}+8 h a_{1} Q_{i}^{k}\right)-\frac{k p}{4} Q_{i-1}^{k}\left(r_{2}-8 h a_{1} Q_{i}^{k}\right),
\end{gathered}
$$




$$
\begin{gathered}
T_{i-1}^{k}=-2 k p Q_{i}^{k}+4 k^{2} c_{1} Q_{i}^{k}+8 h b_{1} Q_{i}^{k}+\frac{k^{2}}{2} S_{i-1}^{k}\left(r_{2}-8 h a_{1} Q_{i}^{k}\right) \\
+\frac{k}{4} p Q_{i+1}^{k}\left(r_{1}+8 h a_{1} Q_{i}^{k}\right)-\frac{3 k p}{4} Q_{i-1}^{k}\left(r_{2}-8 h a_{1} Q_{i}^{k}\right), \\
T_{i}^{k+1}=2 k R_{i}^{k}-8 k p^{2} b_{2} R_{i}^{k}+4 k^{2} c_{2} R_{i}^{k}+\frac{k^{2}}{2} S_{i}^{k+1}\left(1+8 k a_{2} R_{i}^{k}\right) \\
+\frac{3 k}{4} R_{i}^{k+1}\left(1+8 k a_{2} R_{i}^{k}\right)-\frac{k}{4} R_{i}^{k-1}\left(1-8 k a_{2} R_{i}^{k}\right), \\
T_{i}^{k-1}=-2 k R_{i}^{k}+8 k p^{2} b_{2} R_{i}^{k}+4 k^{2} c_{2} R_{i}^{k}+\frac{k^{2}}{2} S_{i}^{k-1}\left(1-8 k a_{2} R_{i}^{k}\right) \\
+\frac{k}{4} R_{i}^{k+1}\left(1+8 k a_{2} R_{i}^{k}\right)-\frac{3 k}{4} R_{i}^{k-1}\left(1-8 k a_{2} R_{i}^{k}\right), \\
T_{i}^{k}=-8 k^{2} c_{1} Q_{i}^{k}-8 k^{2} c_{2} R_{i}^{k}+4 k^{2} S_{i}^{k}-k p Q_{i+1}^{k}\left(r_{1}+8 h a_{1} Q_{i}^{k}\right)+k p Q_{i-1}^{k}\left(r_{2}-8 h a_{1} Q_{i}^{k}\right) \\
-k R_{i}^{k+1}\left(1+8 k a_{2} R_{i}^{k}\right)+k R_{i}^{k-1}\left(1-8 k a_{2} R_{i}^{k}\right),
\end{gathered}
$$

Where

$$
\begin{array}{llll}
\lambda_{1}=-\left(L_{2}+L_{3}+L_{4}\right) \quad, & \lambda_{2}=-\left(L_{2}-L_{3}+L_{4}\right), & \lambda_{3}=6+2 L_{2}+2 L_{4}, \\
\lambda_{4}=-\left(L_{1}-2 L_{3}-2 L_{4}\right) & , \quad \lambda_{5}=-\left(L_{1}+2 L_{3}-2 L_{4}\right), & \lambda_{6}=-12+2 L_{1}-4 L_{4}, \\
\lambda_{7}=\left(L_{2}-L_{3}-L_{4}\right) \quad, & \lambda_{8}=\left(L_{2}+L_{3}-L_{4}\right) \quad, \quad \lambda_{9}=6-2 L_{2}+2 L_{4},
\end{array}
$$

If we put above operators in (4.1) we arrive to a system of equations in which the corresponding matrix is tri-diagonal. We can solve this system with well-known iterative methods such as Krylov subspace methods.

\section{5-Numerical experiment}

In this section, we present a numerical example to show the computational efficiency of the preconditioning methods introduced in Section 2. Our initial guess is the zero vector and the iterations are stopped when the norm of relative residual is less than $10^{-6}$. In following Tables, We show the iteration number without using preconditioner by "no pre". The computations have been done on a P.C. with Corw 2 Pue $2.0 \mathrm{Ghz}$ and 1024 MB RAM. We consider hyperbolic differential equation

$$
\mathrm{u}_{\mathrm{tt}}=\mathrm{u}_{\mathrm{xx}}+\mathrm{u}_{\mathrm{x}}+\mathrm{u}_{\mathrm{t}}
$$

subject to appropriate initial and Dirichlet boundary conditions (1-2,1-3), where

$$
\mathrm{u}(\mathrm{x}, \mathrm{t})=\exp (2 \mathrm{x}+3 \mathrm{t}) \text {. }
$$

We discretized equation (5.1) by using compact high-order approximation. We show the iteration count of different Krylov subspace methods in combination various preconditioning in Tables 2-6. When mesh size $h$ is finer, we encounter break down by 
using direct preconditioners while BLAGE preconditioners work quite well. In Figures 15, comparison of convergence behaviors are shown. Also, In Fig. 6, 7, for sample we show distribution of eigen-values ADI and BLAGE preconditioners that $\mathbf{M}_{1}, \mathbf{M}_{2}$ are left and right preconditioners respectively. It is obvious that the distribution of eigen-value BLAGE preconditioner is regular than ADI.

Number of iterations with GMRES method

\begin{tabular}{|c|c|c|c|c|c|c|}
\hline $\mathrm{N}$ & no pre & Jacobi & SOR & SSOR & ADI & BLAGE \\
\hline $1 / 20$ & 147 & 131 & 72 & 53 & 33 & 63 \\
$1 / 40$ & 350 & 335 & 170 & 144 & 48 & 183 \\
$1 / 60$ & 564 & 530 & 273 & 318 & 220 & 294 \\
$1 / 80$ & 770 & 724 & Nun & Nun & 405 & 455 \\
\hline
\end{tabular}

Number of iterations with CGS method

\begin{tabular}{|c|c|c|c|c|c|c|}
\hline $\mathrm{N}$ & no pre & Jacobi & SOR & SSOR & ADI & BLAGE \\
\hline $1 / 20$ & 162 & 155 & 82 & 57 & 52 & 79 \\
$1 / 40$ & 621 & 878 & 417 & 637 & 114 & 369 \\
$1 / 60$ & 1232 & Nun & Nun & Nun & Nun & 912 \\
$1 / 80$ & 2003 & Nun & Nun & Nun & Nun & 1430 \\
\hline
\end{tabular}

Number of iterations with QMR method

\begin{tabular}{|c|c|c|c|c|c|c|}
\hline $\mathrm{N}$ & no pre & Jacobi & SOR & SSOR & ADI & BLAGE \\
\hline $1 / 20$ & 154 & 143 & 102 & 60 & 45 & 81 \\
$1 / 40$ & 553 & 605 & 407 & 409 & 93 & 309 \\
$1 / 60$ & 1113 & Nun & Nun & Nun & Nun & 781 \\
$1 / 80$ & 1930 & Nun & Nun & Nun & Nun & 1356 \\
\hline
\end{tabular}

Number of iterations with BiCG method

\begin{tabular}{|c|c|c|c|c|c|c|}
\hline $\mathrm{N}$ & no pre & Jacobi & SOR & SSOR & ADI & BLAGE \\
\hline $1 / 20$ & 154 & 148 & 103 & 61 & 44 & 82 \\
\hline
\end{tabular}


A. Golbabai and M.M. Arabshahi/ TJMCS Vol .3 No.1 (2011) 21-34

\begin{tabular}{|c|c|c|c|c|c|c|}
$1 / 40$ & 553 & 608 & 486 & 405 & 91 & 309 \\
$1 / 60$ & 1126 & Nun & Nun & Nun & Nun & 803 \\
$1 / 80$ & 1922 & Nun & Nun & Nun & Nun & 1504 \\
\hline
\end{tabular}

Number of iterations with BiCGSTAB method

\begin{tabular}{|c|c|c|c|c|c|c|}
\hline $\mathrm{N}$ & no pre & Jacobi & SOR & SSOR & ADI & BLAGE \\
\hline $1 / 20$ & 369 & 291 & 96 & 67 & 54 & 83 \\
$1 / 40$ & 1067 & 1265 & 584 & 917 & 111 & 499 \\
$1 / 60$ & 2169 & Nun & Nun & Nun & Nun & 1381 \\
$1 / 80$ & 3503 & Nun & Nun & Nun & Nun & 2132 \\
\hline
\end{tabular}
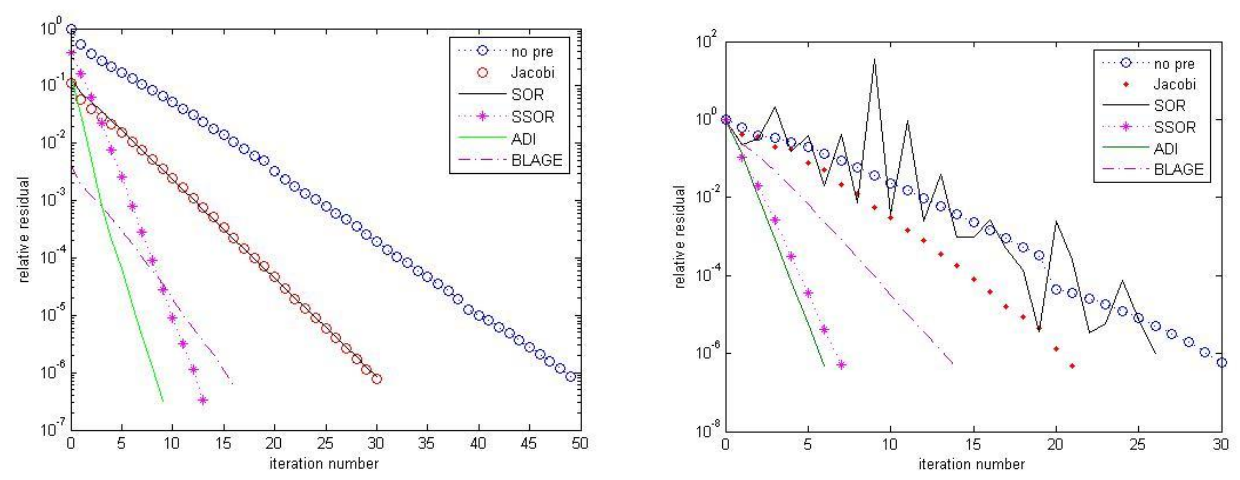

Comparison of convergent behavior of GMRES (left) and CGS (right) methods
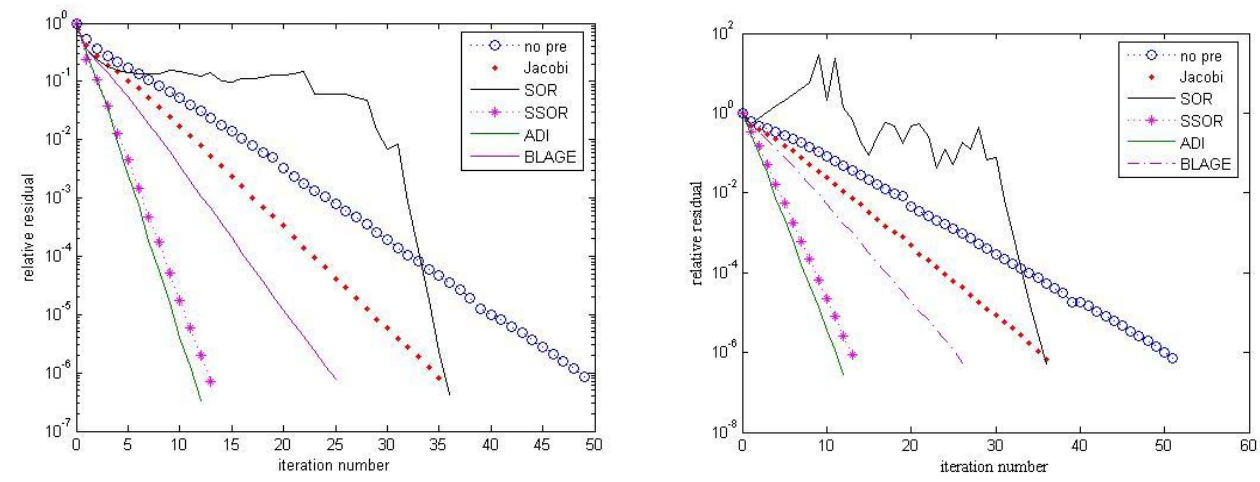

Comparison of convergent behavior of QMR (left) and BiCG (right) methods 
A. Golbabai and M.M. Arabshahi/ TJMCS Vol .3 No.1 (2011) 21-34

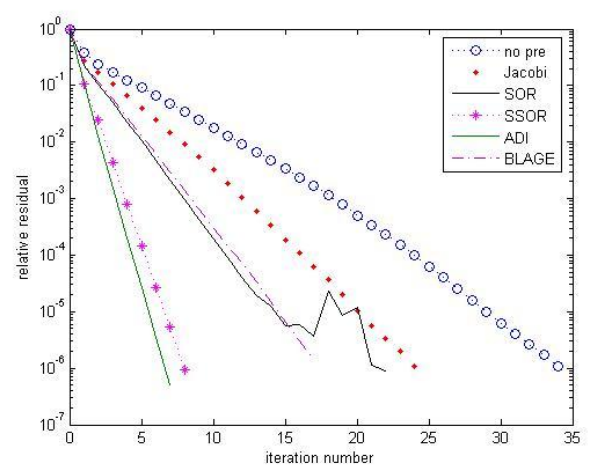

Comparison of convergent behavior of BiCGSTAB method
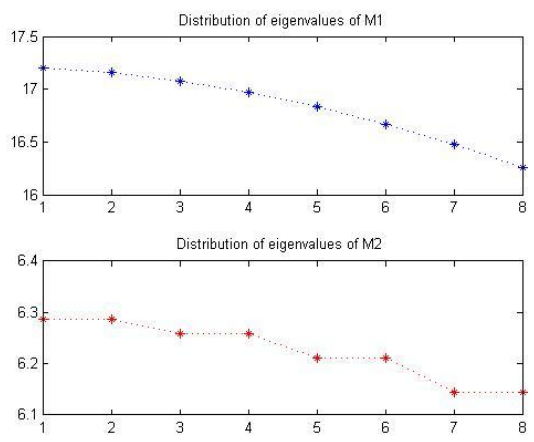

Distribution of eigenvalues in ADI Preconditioner
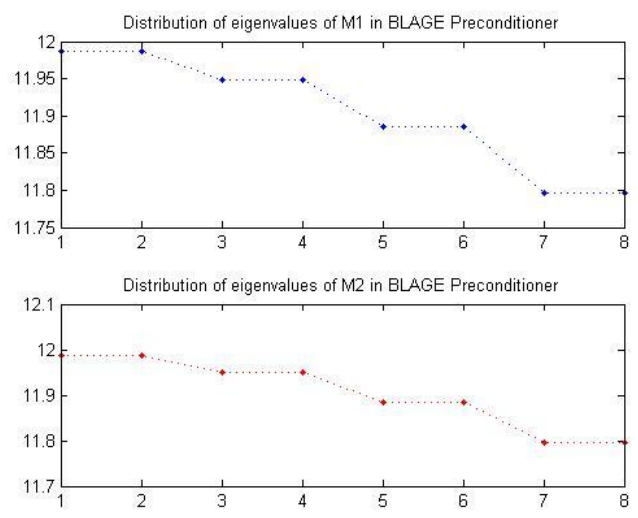

Distribution of eigen-values in BLAGE Preconditioner

We see that we obtain less iteration number with using ADI and SSOR preconditioners but SSOR preconditioner needs more computing time than other preconditioners. Also, we saw that using ADI and BLAGE preconditioner we save in computing time. It is seen that when the condition number is high, the ADI and SSOR preconditioner do not work very well but in well-conditioned problems the iteration number of the BLAGE, SSOR preconditioners is less and the iteration number of the Jacobi and SOR preconditioners is more. We found when mesh size is finer, the QMR, BiCG, CGS and BiCGSTAB methods in composition preconditioners don't work very well but with using GMRES method in 
composition preconditioners, we get less iteration number than other preconditioned Krylov subspace methods. Also, preconditioned GMRES method has regular convergence behavior.

\section{6-Conclusions}

Here, we compared the different preconditioners in non-symmetric systems for hyperbolic equation. From Tables and Figures, we see that although all the methods seem to work well with BLAGE preconditioning using GMRES gives the fastest convergence. Also, the computing time of BLAGE preconditioner is less than other preconditioners. So we propose using BLAGE preconditioner because of less computing time and the less iteration numbers. We propose using the parallel machines for better comparison of block preconditioners because the BLAGE and ADI preconditioners can be employed in parallel environment where the preconditioning operations can be divided into several sub-problems which can be run in parallel [3].

\section{References:}

1. O. Axelsson, Iterative Solution Methods, Cambridge University Press, New York, 1996.

2. R. Barrett, M. Berry, T. F. Chan, J. Demmel, J. Donato, J. Dongarra, V. Eijkhout, R. Pozo, C. Romine. and H. Van der Vorst, Templates for the solution of linear systems: Building blocks for iterative methods, SIAM, 1994.

3. M. Bhuruth and D. J. Evans, Block alternating group explicit preconditioning (BLAGE) for a class of fourth order difference schemes, Intern. J. Comput. Math., 63, 1997, $121-136$.

4. M. Bruaset, A survey of preconditioned iterative methods, Longman Scientific and Technical, UK, 1995.

5. M. Dehghan and S.M. Molavi-Arabshahi, Comparison of preconditioning techniques for solving linear systems arising from the fourth-order approximation of the three-dimensional elliptic equation, Applied Mathematics and Computation, 184, 156-172, 2007.

6. D. J. Evans and M. Sahimi, The alternating group explicit (AGE) iterative method to solve parabolic and hyperbolic partial differential equations in: Annual Review of Numerical Fluid Mechanics and Heat Transfer 11, C.L. Tien and T.C. Chawla, eds., Hemisphere Pub. Co., USA, 1989, 283-390. 
7. D. J. Evans and W. S. Yousif, The block alternating group explicit method (BLAGE) for the solution of elliptic difference equations, Intern. J. Comput. Math., 22, 1987, 177-185.

8. L. C. Evans, Partial Differential Equations, American Mathematical Society Providence, Rhode Island, 1999.

9. R. W. Freund and N. M. Nachtigal, An implementation of the QMR method based on coupled two-term recurrences, SIAM J. Sci. Statist. Comput., 15, 1994, 313-337.

10. Davod Khojasteh Salkuyeh, On the preconditioning of the block tri-diagonal linear system of equations, J. Appl. Math. Comput., 28, 2008, 133-146.

11. M.H. Koulaei and F. Toutounian, On computing of block ILU preconditioner for block tridiagonal systems, Journal of computational and applied mathematics, 202, 2007, 248-257.

12. S. Ma. and Y. Saad, Block-ADI preconditioners for solving sparse non-symmetric linear systems of equations, in: Numerical Linear Algebra, L. Reichel, A. Ruttan and R. S. Varga, editors, Walter de Gruyter and Co., 1993, 165-178.

13. R.C. Mittal and A.H. Al-Kurdi, An Efficient Method for Constructing an ILU Preconditioner for Solving Large Sparse Non-symmetric Linear Systems by the GMRES Method, Computers and Mathematics with Applications, 45, 2003, 1757 1772.

14. R.K. Mohanty and M.K. Jain and Kochurani George , On the use of high order difference methods for the system of one space second order nonlinear hyperbolic equations with variable coefficients, Journal of Computational and Applied Mathematics, 72, 1996, 421-431.

15. S.M. Molavi-Arabshahi and M. Dehghan, Preconditioned techniques for solving large sparse linear systems arising from the discretization of the elliptic partial differential equations, Applied Mathematics and Computation, 188, 2007, 13711388.

16. D. W. Peaceman and H. H. Rachford, The numerical solution of parabolic and elliptic differential equations, J. Soc. Indust. Appl. Math, 3, 1955, 28-41.

17. G. Pini and G. Zilli, Preconditioned iterative algorithms for large sparse unsymmetric problems\}, Numerical methods for partial differential equations, 5 , $1989,107-120$. 
A. Golbabai and M.M. Arabshahi/ TJMCS Vol .3 No.1 (2011) 21-34

18. Y. Saad, Iterative Methods for Sparse Linear Systems, Second Edition, PWS Publishing Company, Boston, 2000.

19. Y. Saad and M. H. Schultz. GMRES, A generalized minimal residual algorithm for solving non-symmetric linear systems, SIAM. J. Sci. Statist. Comput., 7, 1985, 856869.

20. G. L. G. Sleijpen and R. F. Diederik, BICGSTAB(l) for linear equations involving unsymmetric matrices with complex spectrum, Electronic Transactions on Numerical Analysis, 1, 1993, 11-32.

21. P. Sonneveld, CGS, a fast Lanczos-type solver for non symmetric linear systems, SIAM. J. Sci. Statist. Comput., 10, 1989, 36-52.

22. S. Sundar and B,K. Bhagavan, CGS, Comparison of krylov subspace methods with preconditioning techniques for solving boundary value problems, Computers and mathematics with applications, 38, 1999, 197-206.

23. R. S. Varga, Matrix Iterative Analysis, Prentice Hall, Englewood Cliffs, New Jersey, 1962.

24. H.A. Van der Vorst, Iterative Krylov Subspace Methods for Large Linear Systems, Cambridge University Press, Cambridge, 2003.

25. D. M. Young, Iterative Solution of Large Linear Systems. Academic Press, New York, 1971. 